\title{
Promoting Metalinguistic and Metamathematical Reasoning in Proof-Oriented Mathematics Courses: a Method and a Framework
}

\author{
Paul Christian Dawkins ${ }^{1}$ (D) Kyeong Hah Roh $^{2}$
}

Published online: 15 March 2016

(C) Springer International Publishing Switzerland 2016

\begin{abstract}
Students' apprenticeship into proof-oriented mathematical practice requires that they become aware of a range of conventions and assumptions about mathematical language, reference, and inference that rarely appear as explicit components of the content of undergraduate courses. Because these matters of interpretation are often preconscious for students and taken for granted by instructors, students may fail to apprehend the problems at hand and thus misunderstand the mathematical community's solutions. So, we argue that proof-oriented instruction must simultaneously help students understand problems of ambiguity and reference - which requires them to engage in what we call metalinguistic and metamathematical reasoning - as well as provide some informal language for discussing possible solutions to these problems. We provide three illustrative episodes from real analysis classrooms to highlight our common way of framing this class of learning challenge and the pedagogical difficulties they entail. In each case, we highlight the use of analogies as a method of helping students apprehend a problem of mathematical language, reference, or inference and discuss possible solutions. This paper's contributions are thus both practical and theoretical: the instructional method is intended to serve as one possible approach to training students in mathematical conventions and assumptions, and it helps demonstrate our theoretical perspective on this class of teaching and learning phenomena.
\end{abstract}

Keywords Metamathematical learning · Metalinguistic learning · Analogies · Logic · Proof-oriented instruction $\cdot$ Real analysis

Paul Christian Dawkins

pdawkins@niu.edu

1 Department of Mathematical Sciences, Northern Illinois University, DeKalb, IL 60115, USA

2 School of Mathematical and Statistical Sciences, Arizona State University, Tempe, AZ 85287, USA 
Proof-oriented mathematical practice has long been marked by precise and unambiguous language and reasoning. Euclid's Elements served as the paradigm of careful, sequential, and logical argument for millennia. Toward the end of the 19th century, critical questions of mathematical consistency led to further formalization and systematization of the logic and language of mathematical practice. This means that students in proof-oriented mathematical classrooms must acculturate to long-standing practices of language and reasoning (Durand-Guerrier 2008; Epp 2003, 2009), many of which they might not have occasioned to fully need or comprehend. Even among advanced mathematicians, formalizations of linguistic or argumentative practice often arose only after a long period of pre-formalized use. Mathematicians used calculus for several centuries before they constructed the complicated limit apparatus now used to define most of the key ideas therein (Tall 2013). Also, mathematicians constructed proof for millennia before the modern, truth-functional logic of conditionals was formalized. In this paper, we address the particular challenge university faculty face in simultaneously trying to invite students to learn proof-oriented mathematics while attending to such metamathematical (e.g. quantification and reference) and metalinguistic (systematizing language use) issues essential to the fidelity of formalized mathematical theory and practice. By further specifying the problems that render this dimension of teaching so challenging, we lay the foundation for describing one useful method of addressing these problems pedagogically in proof-oriented courses. We then provide a theoretical frame for how this method helps alleviate the learning difficulties described by supporting students' enculturation to the conventions of proof-oriented mathematical practice.

In anticipation of some of the teaching examples that we provide in this article, we first characterize several major barriers to students' acculturation to mathematical proving from the mathematics education and psychological literature. We frame these barriers as matters of metalinguistic and metamathematical reasoning. We use the term metalinguistic (ML) reasoning for practices of organizing and systematizing the language used to speak about objects, properties, and relationships. The learning challenges we investigate have conventionally been characterized as matters of logic, but as Toulmin (1958) argues, that term is often used with conflicting models for what it attempts to describe. Unlike much psychological literature, we do not intend to investigate the implicit logic of people's naïve thought, but rather we investigate students' learning about the problems that logic was developed to solve. Thus, we frame such learning as engaging students in ML reasoning because we endeavor to guide students to 1) perceive problems of mathematical language, 2) develop some (likely informal) language for discussing alternative solutions by which they can 3) evaluate and select among such solutions. We use the term metamathematical (MM) reasoning to refer to students' engagement in organizing and systematizing mathematical assumptions, interpretations, and values pertaining to types of mathematical objects such as examples, definitions, statements, proofs, etc. As with ML reasoning, we perceive MM reasoning requires 1) recognizing a problem pertinent to their mathematical activity, 2) developing informal language to discuss possible solutions, and 3) selecting practices compatible with mathematical norms. We do not intend in this paper to provide comprehensive tools for promoting ML and MM reasoning, but rather we present tools that have helped us address particular challenges in the teaching and learning of real analysis. We offer our instructional method as a possible tool for 
meeting these challenges, and do not claim that it is the panacea for all such difficulties. We do, though, anticipate that our way of framing these instructional challenges will help fruitfully characterize and address other related teaching and learning issues in proof-oriented mathematics classrooms.

\section{The Problems of ML and MM Reasoning Processes}

In this section, we survey pertinent research on students' linguistic and mathematical activity in mathematics. We find ample evidence that students need support in adapting to mathematical conventions of proving, but without guidance students may often be unaware of the sources of these difficulties. First, we describe some aspects of mathematical practice that accomplish the ML goal of disambiguation. Students often use mathematical language in ways contrary to the conventions adopted by the mathematical community, indicating they have not yet had occasion to disambiguate key mathematical meanings and linguistic forms (i.e., the relations between semantic and syntactic systems in mathematics). Second, we consider some MM conventions of defining and inference essential to the generalizability of mathematical proving. While there is some relation between psychological reasoning and formalized proving, research on students' and mathematicians' reasoning suggests that people use a variety of tools for drawing inferences, only some of which are fully compatible with the standards of mathematical proof (e.g. Durand-Guerrier 2008). As with mathematical language, students often fail to adhere to the mathematical conventions of proof that are used to conform argumentation to the community's standards of precision and generality.

\section{Disambiguation and Systematization of Language}

Mathematicians express the vast majority of proof-oriented mathematics through mathematical statements. For us this includes mathematical axioms, definitions, theorems, and much of mathematical proof. Even examples often appear in conjunction with statements of the form "[Mathematical object X] is an element of [the mathematical category characterized by property $\mathrm{P}(\mathrm{X})]$." As such, mathematicians must maintain the sense that mathematical statements carry mathematical information in a faithful and precise way. Accordingly they expend much effort reducing ambiguity and pursuing specificity in their language (Durand-Guerrier et al. 2012). For instance, articles such as "the" must be accompanied by proof that the specified object is unique and mathematicians are quick to acknowledge when something is an "abuse of notation." Many conventions of mathematical language exist for the purpose of uniquely matching linguistic forms (syntactic) to particular mathematical meanings (semantic). When there are multiple meanings possible, mathematicians make meaning more explicit or develop conventions for assigning distinct statements to distinct meanings. Such practices of disambiguation constitute an important mathematical form of ML reasoning.

One common example of disambiguation pertinent to real analysis arises in multiply quantified statements, which includes most limit definitions. A common definition for sequence convergence is, "a sequence $\left\{a_{n}\right\}$ of real numbers converges to a real number $L$ if $\forall \varepsilon>0, \exists N \in \mathbf{N}$ such that $\forall n>N,\left|a_{n}-L\right|<\varepsilon$." Previous mathematics education 
studies show that students often interpret such statements in ways unintended by their instructors (Burn 2005; Cory and Garofalo 2011; Cottrill et al. 1996; Dubinsky and Yiparaki 2000; Durand-Guerrier and Arsac 2005; Epp 2003; Piatek-Jimenez 2010; Roh 2009, 2010; Swinyard 2011). While this sequence convergence definition is often called a "for-every, there-exists" statement $(\forall \exists)$, other multiply quantified statements introduce the quantifiers in the order "there-exists, for every" $(\forall \exists)$. The definition of a sequence being bounded is an example: "a sequence $\left\{a_{n}\right\}$ of real numbers is bounded if $\exists M \in \mathbf{R}$ such that $\forall n \in \mathbf{N},\left|a_{n}\right|<M$." In mathematical practice, the order of quantifiers embeds crucial information about the intended meaning of the statement. In the example of sequence convergence, since $\varepsilon$ appears first, it must be independent of (or cannot be assigned a value in terms of) $N$, which appears later (Roh and Lee 2011). However, the particular tail of the sequence specified by the index $N$ must satisfy conditions relative to the chosen $\varepsilon$-neighborhood of the limit $L$, so the existentially quantified term $N$ often depends upon - or is a function of $-\varepsilon$ (Durand-Guerrier and Arsac 2005). So the existentially quantified $N$ in the definition of sequence convergence does not refer to a single value globally, but rather (at least) one value per $\varepsilon$. Alternatively, the existentially quantified term $M$ in the definition of bounded sequence is understood to correspond to every value of $n$, meaning that it is independent thereof. So the definition of bounded sequence needs only refer to one value of $M$ globally.

The "dependence rule" and "independence rule" that mathematicians use to interpret multiply quantified statements are not written explicitly in the statements themselves. They are matters of convention (Dubinsky and Yiparaki 2000). However, these conventions exist for the purpose of disambiguation. Epp (2003) provides a comparable example statement from non-mathematical language. The book of Ecclesiastes in the Bible is often quoted as saying, "There is a time to every purpose under heaven." The intended meaning becomes clear in the context that multiple times are being assigned to multiple purposes rather than all purposes being assigned to a single moment in time (the relative absurdity "Tuesday at 5, I will accomplish all purposes"). However, according to mathematical convention, the time is quantified first and must thereby be independent of the purpose (i.e. a single time for every purpose). This is not necessarily a problem with the Biblical language because in the discursive domain of poetry, ambiguity and reverse syntax are commonplace, if not celebrated. However, mathematicians rightly try to avoid such linguistic practice in their discursive domain. If the definition of sequence convergence had a different meaning, a different set of sequences would converge (Roh 2008).

\section{Students' Difficulty with Disambiguation}

Acknowledging that interpreting such statements is a matter of convention, Dubinsky and Yiparaki (2000) gave students a series of common language and mathematical statements that could be understood as multiply quantified (both $\forall \exists$ and $\exists \forall$ ). The two mathematical examples were identical statements, except for the order of quantification. They found that students often interpreted everyday statements with $\exists \forall$ syntax in ways that mathematicians would associate with $\forall \exists$ syntax. What the authors found notable was that students did not draw any distinction between the statement and their interpretation thereof; in fact, they claimed that the students did not perceive any "interpretation" to be occurring. They said, "It was as though the statement was a 
window from which they were looking out. The students described what they saw looking out the window, but they did not see the window itself" (p. 261).

This observation that students' linguistic interpretations are pre-conscious reveals the first problem of engaging students in ML reasoning: we communicate through language and it is hard to distinguish when we are talking about the meaning intended to be conveyed by language (semantic) rather than the language itself (syntactic). Communication is a cooperative enterprise. In everyday communication, people try to maintain the norm of listening for people's intended meaning "through" their utterances (e.g. "I see what you mean"; Grice 1957; Thompson 2013). To respond to an alternative interpretation of someone's utterance when they intended something else is considered a breach of communicative norms (e.g. "You know what I mean"). If students have not learned to reason about their own language use, then likely they will both be unaware of ambiguity problems in language and lack a metalanguage for addressing such problems.

We frame Dubinsky and Yiparaki's (2000) findings as saying that many of their participants had not problematized their language use, which is the first step toward ML reasoning. The authors point out that very few of the participants in their study appeared able to simultaneously consider multiple meanings for a single statement (i.e. recognize ambiguity). To make students aware of multiple interpretations, the authors engaged students in a game about the given statements where one player provided the existentially quantified term (Player E) and the other provided values for the universally quantified term (Player A). Player A "antagonized" the statement to prove it false and Player E defended it. The onus was upon Player A to win by disproving the statement, or else Player E won. The players played in the order that the terms appeared in the statement, embedding syntax into the game itself. Students usually recognized that switching the order of play reversed which player had a winning strategy. The game helped the students conceive of the two possible interpretations of multiply quantified statements, thereby problematizing syntactic order. Furthermore, the authors noted that some students were able to produce clearer explanations about the meaning of multiply quantified statements in the language of the game than they had done regarding the bare mathematical and everyday statements previously provided.

Other students in Dubinsky and Yiparaki's (2000) study interpreted the relationship between the game and the statements differently. Compatible with the authors' intentions, some students concluded that the different orders of play corresponded to distinct interpretations of the statement such that the game revealed ambiguity that could be resolved by assigning different meanings to two different statements. Other students protested that while the rules of the game led to different outcomes, "That is not in the statement... I mean, because you change it into a game, I saw it as a win or lose, and manipulation" (Dubinsky and Yiparaki 2000, p. 282). This student pointed out that the conventions of interpretation embedded in the rules of the game are not explicitly in the statements themselves, and she viewed the game as being manipulated to help the interviewer win. This reveals a second problem for engaging students in ML reasoning. Since some matters of interpretation are merely conventional (very useful conventions, but conventions none-the-less), how can we help students feel that these conventions are useful or necessary? As mentioned above, differentiating someone's articulation from their meaning feels like a breach of communicative norms. 
Based on their findings, Dubinsky and Yiparaki (2000) endorse the game for instruction, but recommend against making analogies to everyday language to teach mathematical conventions of interpretation. They suggest that mathematics instructors must teach mathematical syntax and the conventions thereof, but this may be best done in mathematical contexts. Many other researchers on the role of technical language use in proof-oriented mathematics instead argue that making analogies with well-chosen everyday statements can support students' adoption of mathematical conventions (Durand-Guerrier and Arsac 2005; Durand-Guerrier et al. 2012; Epp 2003, 2009). We shall return to this matter later regarding our examples of instruction.

\section{Conventions of Reference}

Another prominent value in mathematical practice is generalization. Mathematicians adopt a number of conventions in pursuit of maximally inclusive conclusions. For instance, mathematical language is often abstract as a means toward generality. When a proof refers to a single, abstract object ("Let $\varepsilon>0$ ", "Let $f$ be a continuous function", etc.), then by the principle of universal generalization the claim is assumed proven for any such object in that category (Copi 1954; Durand-Guerrier 2008). The fidelity of this inference depends upon two other important conventions of mathematical reference.

One convention is that mathematical statements are interpreted as closed general statements, meaning that they have a pre-determined scope of reference (DurandGuerrier 2008; Jahnke 2008). A statement is false if it fails for at least one instance within its scope. So the statement "If a function is continuous, then it is differentiable" is false even though it holds true for most of the continuous functions familiar to students. Durand-Guerrier (2008) argues that some students are prone to interpret mathematical statements as open, meaning they interpret the statement via the question "for which instances is this true?" In this interpretation, students implicitly vary the statement's scope of reference (the statement is true given the proper restrictions, but false if considered for the set of all continuous functions). Durand-Guerrier (2008) argues that such open interpretations are useful as they afford students' exploration of further mathematical patterns rather than the mere assignment of truth-values to given statements. Mathematicians often add or relax hypotheses (i.e. vary the scope of reference) to achieve new possible theorems or understand why such alternatives fail.

We do not claim students adopt such interpretations intentionally; as in the case of quantifiers students are likely not aware of their linguistic interpretive process (Stenning and van Lambalgen 2004). Rather, students' interpretations likely arise from their heuristics for testing such claims (Stenning 2002), which may rely heavily on familiar examples. The difficulties entailed in investigating classes by examining particular exemplars leads to the second convention that supports mathematicians' pursuit of generalization, which is maintaining stipulated definitions (Edwards and Ward 2008). Cognitive science research suggests that many people's everyday concepts are constructed around paradigm examples (e.g. Murphy and Hoffman 2012) rather than upon properties that unify the exemplars. As a result, many such categories entail differing degrees of membership (prototypes, borderline examples, and others between). Lexical definitions then merely seek to describe such pre-existing categories (what Edwards and Ward call extracted definitions). Such fuzzy categories would 
undermine the clear reference of mathematical statements and thus the ability to prove claims generally true or false, so the mathematical community avoids this interpretation of mathematical definitions. Mathematical definitions are understood to set forth properties that completely designate a category (stipulated), even if some members of that category conflict with the intuitions from which the category was derived. The convention of stipulated definitions supports the mathematical value of generality by allowing any proof using abstract properties to apply to as-of-yet unimagined examples with a given property (Alcock and Simpson 2002).

In this way, mathematicians take a seemingly circuitous route to prove why examples of differentiable functions are also continuous. They first construct stipulated definitions for the properties perceived. Though these definitions may be descriptive in their psychological generation (De Villiers 1998; Freudenthal 1973), they are stipulated in their mathematical application. Proofs of the statement "If a function is differentiable, then it is continuous" do not attempt to refer to particular examples of functions in the categories, but rather establish that the property defining differentiability entails the property defining continuity. That one property entails the other then generalizes to every function that exhibits the properties. Thus, logical entailment of properties corresponds to a subset relation between the classes of mathematical objects (the class of differentiable functions is a subset of the class of continuous functions). This transposition of properties for exemplars in tandem with closed general interpretation of mathematical claims affords mathematicians' goal of generating general proof of mathematical claims ("All differentiable functions are continuous").

What we are pointing out in this section is not novel or surprising per se. Mathematicians consistently embed these conventions into their ways of speaking and acting. A common metaphor in mathematical parlance is the set or space of mathematical objects. Mathematical objects like functions, numbers, theorems, equations, etc., can all be imagined as points in a space. Conditions and definitions stipulate sets within that space. Implications between conditions or definitions then translate to subset relations via the metaphor. What we are clarifying is that the fidelity of this metaphor intrinsically depends upon these conventions of reference. Statements must be quantified over (refer to) well-defined sets in these spaces.

\section{Student Difficulties with Conventions of Mathematical Reference}

Similar to the conventions of multiple quantification, MM conventions of reference are often imposed in the classroom without being explicitly taught or negotiated. Students' untrained reasoning even in proof-oriented courses often differs from the conventions of mathematical practice. Students may implicitly interpret mathematical definitions as extracted by reasoning primarily about exemplars or elements of their concept images (Alcock and Simpson 2002; Edwards and Ward 2008; Vinner 1991). Ouvrier-Buffet (2006) observed that students who created mathematical definitions enacted different senses of what those definitions should do. Some students' definitions identified examples from non-examples (e.g. an even number ends in $0,2,4,6$, or 8 ) while others sought to construct the object via essential properties (e.g. an even is a multiple of 2). While the former are useful when presented with a given mathematical object, it may not equally support a generalized proof activity through the method of universal generalization. Furthermore, how can students reflect on and negotiate such divergent 
interpretations of these meta-concepts central to mathematics? This returns to our central theme in this paper: Students' MM reasoning processes differ from those of mathematicians, but prompting and facilitating students' $\mathrm{MM}$ reflection is quite challenging.

The matter of reference creates problems in proving as well as defining. Students may reason about a proof in reference to a diagram without maintaining the "arbitrariness" of the chosen instantiation. While mathematics cannot avoid investigating the general through the particular (e.g. Eisenberg and Dreyfus 1994; Font and Contreras 2008; Guzman 2002; Mariotti and Fischbein 1997), helping students navigate this relationship is challenging. For instance, it is not uncommon to phrase a theorem in a conditional form - "If [hypotheses], then [conclusion]" - hiding the implicit quantification - " $\forall$ case in which [hypotheses], [conclusions] occur." However, students may interpret the phrase "If..." as invoking a hypothetical instantiation - "imagine a case where" - rather than a code for universal instantiation - "in any such case that."

For this reason, many studies in mathematics education suggest that instructors should coordinate the teaching of definitions and proof with exploration of a range of possible examples. Coordinating examples and properties encourages students' natural sense of reference (interpreting claims in light of known examples) to attend to a broader range of important exemplars (Dahlberg and Housman 1997; Mason and Watson 2008; Swinyard and Larsen 2012). Specifically, Mason and Watson (2008) emphasize students' awareness of dimensions of possible variation within a mathematical category. As students reason about given statements, we should not necessarily expect them to only reason about the concept definition itself. However, to use examples fruitfully, students must be able to intentionally vary relevant aspects of chosen examples to observe invariances that might generalize (Antonini 2011; Lockwood et al. 2012; Pinto and Tall 2002). Dimensions of possible variation are often revealed by boundary examples, which are members of a category that differ in some significant way from prototypical examples. Unfortunately, students may resist this instructional trajectory and entrench in their restrictive example-based reasoning by monster-barring (Lakatos 1976; Larsen and Zandieh 2008). That is, the students may simply reject counterintuitive examples regardless of the definition. None-the-less, we hold that students should be trained to coordinate the processes of concept stretching (Lakatos 1976) though progressive generalization across boundary examples and reasoning with definitions (Alcock and Simpson 2002).

\section{Fostering ML and MM Reasoning}

We provide this extensive discussion of linguistic and mathematical conventions essential to proof-oriented mathematical practice both to clarify the instructional problems upon which this paper focuses and to highlight the intrinsic challenge of talking about language or discussing metamathematics. We operate under the working assumption that mathematical learning should be a process of sense making, such that the understandings that students develop helps solve felt problems in their goal-oriented activity (Brousseau 1997; Freudenthal 1973, 1991). The problem with ML and MM matters of interpretation (and the corresponding conventions of practice) is that we perceive they are often invisible to students and taken-as-obvious by mathematics 
instructors. While mathematics faculty may understand how alternative conventions of language and interpretation undermine mathematical activity, motivating and communicating these matters to students is non-trivial, as the above discussion showed. Furthermore, students' acculturation to these modes of language and interpretation are only necessary, but not sufficient, conditions for learning proof-oriented mathematics. Conventions of ML and MM practice are often not explicitly part of the curricular content of undergraduate proof-oriented courses. As such, it is hard to know how and when they should be addressed, if at all. Neither is it clear what mode of introduction will be most effective. To present an initial introduction to mathematical logic (e.g. truth tables, methods of negating compound statements) may provide abstract tools that answer questions that students have not yet asked. To ignore the problems of language and reference may perpetuate faculty and students "speaking a different language." Our central question is: "How can proof-oriented instruction surface ML and MM problems in the course of instruction to help students problematize these issues and develop tools for acting in manners compatible with essential mathematical conventions?"

In this regard we strongly endorse the work of Durand-Guerrier, Epp, and their colleagues (Durand-Guerrier and Arsac 2005; Durand-Guerrier et al. 2012; Epp 2003, 2009) because they present rich activities that problematize linguistic interpretation and MM issues to students. Two very useful contributions of their work are the following principles for instruction. First, students' linguistic and interpretive schemes in the classroom are not misconceptions so much as rational behaviors reflective of the ambiguity in everyday language. In many cases, everyday associations of meaning and linguistic form violate mathematical conventions (Epp 2003). Second, everyday language use and reasoning should not be discounted from classroom instruction because well-chosen instances of common language use and reasoning can serve as paradigms of the conventions that mathematicians adopt more uniformly. Our instructional method relies upon such well-chosen analogies that help students perceive and adopt mathematical conventions of interpretation.

\section{Three Example Analogies for Instruction}

In this section, we provide accounts of three analogies used to help surface and discuss ML and MM conventions in real analysis courses. Each account arose during one of two studies of real analysis instruction conducted by the current authors. These studies each investigated student learning in the context of inquiry-oriented, real analysis instruction (Dawkins 2014; Dawkins and Roh 2011) taught by experienced and expert university faculty. Students in these courses were mathematics majors in their third or fourth years at two large public universities in the Southwestern United States. These courses focused heavily on proving rather than computation. For convenience we shall refer to these two classes as RA1 (taught by Dr. B) and RA2 (taught by Dr. K). The studies were originally conducted separately before the authors embarked on a comparative analysis of the emergent parallels of instructional trends in the two classrooms. The pattern of using analogies for teaching mathematical conventions appeared in both classrooms, motivating a fuller exploration of the nature and efficacy of the practice. This retrospective, comparative analysis fostered the emergence of our ML and MM frame for the learning phenomena in question. We explain why we consider this frame 
as a valuable contribution of the current investigation after we have presented the three illustrative examples of analogies for instruction.

\section{The "White Tigers" Analogy for Existential Quantification"}

RA1 spent several class days early in the semester learning about cardinality of infinite sets. During class, Dr. B presented the proof that the natural numbers $(\mathbf{N})$ have the same cardinality as the even natural numbers $(2 \mathbf{N})$ because there exists a bijection $(1: 1$ and onto function) between them. To guide student thinking about the existence of bijections, the professor presented an analogous definition in a non-mathematical context: "A forest is called special if there exists a white tiger in it." Dr. B was aware that students often questioned the existence of examples with certain properties by focusing on other, more familiar examples. She extended the analogy to possible arguments about whether a particular forest was "special" or not. She asked whether searching all day and seeing only orange tigers would imply that the forest was not special. Beyond simply portraying a "there exists" definition, the analogy and surrounding discussion emphasized several aspects of proof regarding existentially quantified statements:

- One must begin a search for a satisfactory example (white tiger) to prove the statement.

- One cannot search all possibilities (the forest is inexhaustible).

- A single satisfactory example constitutes sufficient verification (one white tiger is sufficient).

- No number of non-examples constitutes sufficient verification against the existence claim (orange tigers are inconclusive evidence).

During a study session Dr. B organized after class, students worked on a homework problem asking them to find a function from $2 \mathbf{N}$ into $\mathbf{N}$ that was injective (1:1) but not surjective (onto). One group, citing the (inverse of the) bijection defined in class, questioned whether such a function exists at all. Hearing the group's reasoning, Dr. B wrote on the board " $\mathrm{T} / \mathrm{F}$ If there exists a bijection $f: A \rightarrow B$, then there does not exist a function $g: A \rightarrow B$ which is injective but not surjective." While her query on the board was in a mathematical register, she verbally referred to the functions in the analogical register: "That's what you told me. I just went into the jungle and found a white tiger, therefore that proves there are no orange tigers." The students appeared satisfied by this refutation of their previous claim and worked to produce an injective but not surjective function.

Much later in the semester, during an interview with one of the authors, the student Edgar reflected on proving that sequences do not converge. He spontaneously called upon the White Tigers analogy to articulate his reasoning.

Int.: You said it is easy to show something doesn't converge, so why did you say that?

\footnotetext{
${ }^{1}$ The White Tigers analogy previously appeared in Dawkins (2009), Shipman (2012), and Shipman and Shipman (2013). 
Edgar: You can show multiple [sub]sequences that converge to multiple things... In general whatever you say, for every, it seems much easier to take, if someone says "For every," and you say, "No, I have got one that doesn't match that," to use as a disproof, or to disprove something. Whereas when you make a claim about "for every" it is usually very difficult to say. You know? The white tiger thing. "All the tigers in this forest are orange." "Oh, really? Find them all and bring'em here."

Though the White Tigers analogy was introduced in relation to an existential statement, Edgar applied the analogy accurately to the negation of a universally quantified statement. The class did not discuss "rules" for the negations of mathematical statements such as "The negation of a 'for every $(\forall)$ ' statement is a 'there exists $(\exists)$ ' statement." Edgar used the analogy because he perceived compatibility between the standards of evidence for a proof of an $\exists$-statement and a disproof of an $\forall$-statement in the two mathematical contexts.

\section{Comments on the White Tigers Analogy}

The two conditions for learning we set forth for the instructional method were 1) to make students reflect on ML or MM issues and 2) to provide them with tools for adopting ways of acting consistent with mathematical convention. In what ways does the White Tigers analogy do so? First, we observed that such analogies were very common in Dr. B's parlance throughout the semester (Dawkins 2009, 2012). She used them as a tool to address confusion or for surfacing student misconceptions that she anticipated based on her teaching experience. The issue at hand was students' ability to 1) think about properties of sets defined by functions between them and 2) students' ability to create and classify diverse exemplar functions between two sets. Dr. B's analogy portrayed how the function classified the sets because it classified forests by the presence of white tigers. While the property concerns the sets (having the same cardinality), proofs or disproofs rely upon functions. Thus, we observe that the analogy helped draw students' attention to functions' role in the definition. Furthermore, Dr. B's choice of forests as the tiger space portrayed the set's inexhaustibility. The point she emphasized was that one could not find every possible function (tiger), so one must be careful when claiming a function does not exist. Implicitly, this introduced the common mathematical metaphor of mathematical objects' reference over some example space.

How does this relate to MM reasoning? While the analogy mapped elements of the definition onto the "special forest" definition, the way Dr. B used the analogy induced an implicit metaphor between standards of evidence in mathematics and the analogical domain. The more important work that the metaphor accomplished was providing students with tools for reflecting on the validity of arguments for or against the existence of a particular type of function. Many studies on students' thinking about categories suggest that they tend to reason in terms of familiar exemplars (Alcock and Simpson 2002; Mason and Watson 2008). Similarly, the students during the study session described in this episode struggled to construct a merely injective function because they focused on the bijective function shown in class. Dr. B used the language of the White Tigers analogy to refute the students' tentative argument of non-existence. What she wrote on the board merely revoiced (O'Connor and Michaels 1993) the 
students' claim in the mathematical register. While her response could be said to similarly accomplish the goal of having students reflect on the validity of their argument (MM reasoning), revoicing alone did not provide students with any further tools for evaluating their claim. We observe that aspects of the analogy served as implicit warrants for her refutation of the students' reasoning. She implicitly beckoned to the analogy's portrayal that mathematical spaces tend to be unsearchable, just as one cannot find all tigers in a forest.

The students accepted Dr. B's counterargument and moved on to complete the task. We cannot claim that the students revised their claim only because Dr. B employed the analogy/metaphor since they might have submitted to her hierarchical authority as their professor (Gerson and Bateman 2010). We use this example, however, to portray the pattern of communication featured in this paper. As we argued, the analogy sought to guide students' reflection on matters of argumentation regarding "there exist" definitions (an MM topic). What is more, the analogy provided a language for discussing such arguments. For example, when Edgar was trying to speak about standards of proof and disproof in a different context (an instance of MM language), he called upon the White Tigers analogy to express his thinking. Finally, we value this instructional method because it was "minimally invasive." Dr. B introduced the analogy topically and made recourse to it when she needed, but did not devote extensive time to instruction on the MM topic of proofs and disproofs of existentially quantified statements. Clearly, we do not think this mode of instruction will lead students to a thorough and systematic knowledge of mathematical logic. However, mathematics educators have pointed out that much mathematical proof relies only on a minimal set of logical principles (Durand-Guerrier 2008; Savic 2011). Though we want students to formalize their language use and proving in real analysis, we do not intend for students to develop formal understanding of metamathematics. We instead intend to help students act in ways compatible with mathematical convention. In the next episode, we hope to reveal the same pattern of surfacing and resolving ML and MM issues through the use of topical analogies, specifically regarding the interpretation of multiply quantified statements.

\section{The Mayan Analogy for Multiple Quantification²}

RA2 spent several class days studying the $\varepsilon-N$ definition of the limit of a sequence, its related properties, and proofs of the convergence/divergence of sequences. During class, Dr. K presented an argument from a hypothetical student, Bill, about the convergence of the sequence $\{1 / n\}$ :

Bill's argument. For all $N \in \mathbf{N}$, choose $\varepsilon=\frac{1}{(N+2)}$. Then $\varepsilon>0$ since $N>0$. Let $n=N+1$. Then $n>N$. Also, $\left|\frac{1}{n}-0\right|=\frac{1}{n}=\frac{1}{(N+1)}>\frac{1}{(N+2)}=\varepsilon$. Therefore, $\left\{\frac{1}{n}\right\}$ does not converge to 0 .

The class worked in small groups to evaluate Bill's argument. A group of four students, Amy, Elise, John, and Matt (pseudonyms), easily noticed Bill's argument was invalid because of its false conclusion. When Amy and John insisted that the sequence

\footnotetext{
${ }_{2}$ The Mayan story previously appeared in Roh and Lee (2011).
} 
$\{1 / n\}$ converges to 0 and Elise and Matt agreed with them, these students' attention remained on Bill's mathematical claim (the sequence converges to 0 ) rather than on the structure of his argument.

Later, when the students checked the rest of the statements in Bill's argument, some of them thought they were correct except for the conclusion. The students could not articulate why Bill's argument was mathematically invalid. For instance, Elise expressed suspicion about Bill's negation the $\varepsilon-N$ definition. She said, "Bill's argument didn't seem like a proper negation ... I don't know ... Everything doesn't seem to line up for. Some of it is negated, some of it didn't, and I don't know." She also suggested that the choice of $\varepsilon$ "didn't look right." However, she was unable to critique the argument more specifically. Elise did not show evidence of attending to any "independence" or "dependence" among the variables $\varepsilon, N$, and $n$.

As we stated previously, the "independence rule" is nowhere embedded in the $\varepsilon-N$ definition of the limit of a sequence and may not apply to similar statements in everyday contexts. To help the class reason about the various possible interpretations of multiply quantified statements, Dr. K introduced "the Mayan stonecutter story," presented in Fig. 1. The story provided students with an analogical portrayal of the various interpretations of a $\forall \exists$ statement.

In the same way that the students rejected Bill's argument in the previous task, they immediately noticed the unfairness of the priest in the Mayan stonecutter story. In contrast to their evaluation of Bill's argument, the students recognized more clearly how the craftsman and priest's arguments differed (specifically whether the priest's argument refuted the craftsman's claimed ability). Matt restated the craftsman's claim in quantified language: "for all distances or gaps, there exists an amount of time for which the craftsman can get that distance." Elise negated Matt's interpretation of the

\section{The Mayan Stonecutter Story}

One of the famous Mayan architectural techniques is to build a structure with stones. These stones were ground so smoothly that there was almost no gap between two stones. It was even hard to put a razor blade between them. One day a priest came to a craftsman to request smooth stones.

Craftsman: No matter how small of a gap you request, I can make stones as flat as you request if you give me some time.

Priest: I do not believe you can do it. If I ask you to flatten stones within $0.01 \mathrm{~mm}$, you won't be able to do it.

Craftsman: Give me 10 days, and you will receive stones as flat as within $0.01 \mathrm{~mm}$.

Ten days later, the craftsman made two stones so flat that the gap between them was within 0.01 $\mathrm{mm}$. On the 11 th day, the priest came to see the stones and argued that,

Priest: $\quad$ These stones are not flat within $0.001 \mathrm{~mm}$. What I actually need are stones as flat as within $0.001 \mathrm{~mm}$.

Craftsman: Okay, if you give me 5 more days, I can make the stones as flat as within $0.001 \mathrm{~mm}$.

Five days later, the craftsman made the two stones so flat that the gap between them was within $0.001 \mathrm{~mm}$. On the 16th day, the priest came to see the stones and argued that,

Priest: $\quad$ But these stones are not flat within $0.0001 \mathrm{~mm}$ and I meant $0.0001 \mathrm{~mm}$. You don't have that kind of skill, do you?

If the priest keeps arguing this way, is the priest really fair showing that the craftsman does not have the ability to flatten stones within any margin of error?

Fig. 1 The Mayan stonecutter story (Roh and Lee 2011, p. 694) 
craftsman's claim: "your negation [of the craftsman's claim] is that there exists a distance between them such that for all time you give them, you can't make it or that." Comparing the negation of the craftsman's claim with the priest's argument, Matt found that the order of quantified variables, distance and time, was reversed. He explained, "He [the priest] is flipping them. He is saying for all time, there is - the existence of - a distance that you can't get to." Elise agreed with Matt that the priest's argument was problematic because it reversed the order in which distance and time were determined. Elise said that for the priest to disprove the craftsman's claim, the priest would have to show that "there exists a distance that no matter how much time the craftsman has, he [the craftsman] cannot make that distance." Instead the priest interpreted the claim as: "no matter how much time the craftsman has, there was a gap that he hadn't reached yet." Matt observed that the differing interpretations stemmed from the order in which time and distance were determined.

Flipping those [distance and time] is a big deal because if you're saying "there exists a distance that no matter how much time he has, he can't get to," that's way different from saying "for any times, at this time, there exists one thing that doesn't work." Well, that doesn't tell us a whole lot about the negation of the original statement, but tells us what happens at that one time. But then the distance is dependent on whatever time.

The students' discussion reveals that the Mayan stonecutter story shifted their attention to the order of determination and dependence of the two quantities. The students quickly identified the relevant structural differences between the craftsman's claim of skill and the priest's counterargument. The priest's argument did not negate the claim of the craftsman's skill because he reversed the order of the quantities imposing a single time for all distances rather than a distinct work time for each distance between stones.

Furthermore, the students quickly used the Mayan stonecutter story to identify a flaw in Bill's argument. Dr. K did not draw an analogy between Bill's argument and the priest's argument, but the students recognized the similarity. Both arguments assume the first quantity may depend upon the second in a multiply quantified statement. The students decided that Bill's chosen $\varepsilon$ that depended upon $N$ led to the false conclusion. For instance, Matt said:

In the first problem [Bill's argument] where we're looking at them, where you have three different quantified statements. And you're reversing [negating] all three of them [' $\forall \varepsilon>0,{ }^{\prime} ' \exists N \in \mathbf{N},{ }^{\prime} \&$ ' $\forall n>N$ '] that we have, but then we moved one out of order [...] When I first saw it, I thought "Oh! They negated them correctly." Well, as we look into them closer, they didn't really, you know? They negated all of the quantifiers. They just took them out of order and changed the dependencies relationship [...] It [Bill's argument] is kind of cool example. [laughs]

Finally, Dr. K wanted to test whether the students' newfound awareness of the mathematical conventions for multiply quantified statements was robust. They may have only interpreted Bill's argument in terms of the craftsman's reasoning because the 
two activities were presented sequentially in class. So, 2 weeks ${ }^{3}$ later after the class discussed the definition of Cauchy sequences, Dr. K introduced Statement 1 about Cauchy sequences to the class and asked to prove or disprove the statement.

Statement 1: If a sequence $\left\{a_{n}\right\}$ in $\mathbf{R}$ is a Cauchy sequence, ${ }^{4}$ then for any $\varepsilon>0$, there exists $N \in \mathbf{N}$ such that for all $n>N,\left|a_{n}-a_{n+1}\right|<\varepsilon$.

The students in this group previously quoted determined Statement 1 as a true statement. They also outlined how to prove the statement by substituting $n+1$ for $m$ in the inequality $\left|a_{n}-a_{m}\right|<\varepsilon$ of the definition of a Cauchy sequence. Dr. $\mathrm{K}$ then introduced Ben's argument (also a hypothetical student) and asked the students to evaluate if it appropriately disproved Statement 1.

Ben's argument: Consider $a_{n}=\frac{1}{n}$ for any $N \in \mathbf{N}$. Since the sequence $\left\{a_{n}\right\}$ is convergent to 0 , it is a Cauchy sequence. Let $\varepsilon=\frac{1}{(N+1)(N+2)}$ for all $N \in \mathbf{N}$. Let $n=N+1$. Then $n>N . \quad$ But $\left|a_{n}-a_{n+1}\right|=\left|a_{N+1}-a_{N+2}\right|=\left|\frac{1}{N+1}-\frac{1}{N+2}\right|=\frac{1}{(N+1)(N+2)}=\varepsilon \geq \varepsilon$. Therefore, Statement 1 is false.

The group insisted that Ben's argument does not disprove Statement 1, regardless of the truth of Statement 1. Unlike their initial objections to Bill's argument, which focused on its false conclusion, the students attended to how Ben's argument related the quantified variables. They pointed out that choosing an $\stackrel{a}{\text { that }}$ depended on $N$ was problematic, saying:

Matt: If they're trying to disprove which is what they're doing, they need the existence of one $\varepsilon$. Well, if that one $\varepsilon$ is dependent on all possible $N-$

Elise: Then it changes.

Matt: Yeah, it seems to be ... well, then, it's backward from where the quantifiers are.

Elise: Yeah, exactly. [...] $E[\varepsilon]$ is dependent on the $N$. And that isn't allowed because where the quantifiers are $N$ comes after $\varepsilon$. So $\varepsilon$ must happen before $N$ does. $[\ldots]$

Matt: Yeah, [...] it switches the order. It creates dependency [of $\varepsilon$ on $N]$.

Elise: Yeah.

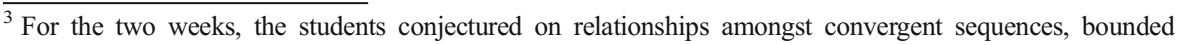
sequences, monotone sequences, and Cauchy sequences. The classes were devoted to discuss how to verify the students' conjectures. For the two weeks, the Mayan stonecutter story was not mentioned at all by Dr. K or by the students.

${ }^{4}$ The definition of Cauchy sequence introduced to the students in the class is as follows: A sequence $\left\{a_{n}\right\}$ in $\mathbf{R}$ is a Cauchy sequence if for any $\varepsilon>0$ there exists $n \in \mathbf{N}$ such that for all $n, m \in \mathbf{N}$ with $n, m>N,\left|a_{n}-a_{m}\right|<\varepsilon$.
} 
Matt: And the brick or whatever. (laughs)

Elise: Rock carver.

So, not only did the students clearly attend to dependence and order in Statement 1 and in Ben's argument, but they also made reference to the Mayan stonecutter story as a paradigm of the argumentative flaw.

\section{Comments on the Mayan Analogy}

As the beginning of the episode portrays, students were often unaware of matters of dependence or independence in multiply quantified statements. At the level of mathematical claims, the students in RA2 recognized Bill's argument was spurious because it had a false conclusion. However, they lacked the MM and ML tools for recognizing and articulating the flaw in the argument. Bill's argument assumes an interpretation of the sequence convergence definition incompatible with mathematical convention, though many students share that interpretation. Even those students in the class who held the conventional interpretation were unable to identify and differentiate the two views. So, Bill's argument problematized linguistic interpretation of mathematical statements, but did not provide tools for further ML reasoning.

The Mayan stonecutter story provided a voice to both interpretations simultaneously so that students could contrast the craftsman and priest's interpretations. This was a form of revoicing in the analogical register. We perceive that a novel element the story introduced was the implicit metaphor between chronological order and dependence. It is common to hold that events that happen later chronologically may "depend" upon earlier ones and earlier events must be independent of later ones. By the stonecutter and priest determining their requirements for work time and gap distance in time, students perceived how order induced dependence (and independence) relations. The contractual aspect of the story also induced a deontic aspect to the arguments, which has been shown in psychology research to (positively) affect how students interpret claims and their negations (Evans 2005; Stylianides and Stylianides 2008). The analogy thereby helped students distinguish the two interpretations and select the one adopted by mathematical convention.

Furthermore, the Mayan stonecutter story introduced language the students used to discuss and critique the arguments in terms of "order" and "dependence." Later, when they examined Ben's argument about Cauchy sequences, the group displayed continued awareness of the order's significance for dependence and independence. They also referenced the Mayan analogy both explicitly and implicitly. Explicitly they cited the characters ("rock carver"). Implicitly, they borrowed the metaphor between temporal order in the statement and chronological order. While Matt cited "where the quantifiers are," Elise translated the claim saying, " $\varepsilon$ must happen before $N$ does." In this way, the Mayan stonecutter story satisfied both of our instructional goals: 1) helping students reflect on $\mathrm{ML}$ and $\mathrm{MM}$ issues and 2) providing a language for discussing them productively. Like the White Tigers analogy, it was minimally intrusive in the course of instruction, but quite useful for helping students overcome common misconceptions and adopt standard mathematical conventions of language and argumentation. The next and final episode provides a third analogy/metaphor pair that we claim helped surface 
and resolve ML and MM issues, particularly regarding the relationship between mathematical examples and definitions.

\section{The Penguin and Platypus Analogies for Atypical Exemplars}

As advocated by research on defining in proof-oriented mathematics, Dr. B used a large, diverse set of examples to guide students' reasoning about constructing definitions and theorems. She paid particular attention to the common student misconception that only monotonic sequences tend to infinity. ${ }^{5}$ As was her usual practice, she asked the students to consider what it meant for a sequence to "tend to infinity" or "diverge to infinity." After exploring some monotonic examples, Dr. B introduced the sequence $\{1,1,2,1,3,1,4 \ldots\}$ initially calling it "Platypus." She asked the class whether this sequence tended to infinity because it certainly did not act exactly like the previous examples. She explained that the platypus is a strange animal because it has hair like a mammal, but it also lays eggs unlike other mammals. She pointed out that scientists could choose to classify the platypus as a mammal because it has hair or not to because it lays eggs, but whatever choice they made needed to be consistent. She then explained that animals with hair are mammals and so the platypus is a mammal.

Dr. B then introduced the example $\{2,1,4,3,6,5,8,7 \ldots\}$ asking whether it tended to infinity. When students assented that it did, Dr. B renamed the second sequence Platypus and the first Penguin. She explained that penguins look like they have fur, but actually have feathers. By these analogies, she asserted that the first sequence $\{1,1,2$, $1,3,1,4 \ldots\}$ looked like it tended to infinity, but was not in the category. The second example $\{2,1,4,3,6,5,8,7 \ldots\}$ does tend to infinity, but in an odd way (nonmonotonic).

Next, Dr. B helped the class produce a definition for $\lim _{\mathrm{n} \rightarrow \infty} x_{n}=\infty$ modeled after their definition of sequence convergence. After producing a proof that the sequence $\{\ln n\}$ satisfies their definition of a sequence tending to infinity, she provided the following true/false questions for the students to discuss in groups and then as a class (note that if $\lim _{n \rightarrow \infty} x_{n}= \pm \infty$, then the sequence "properly diverges"):

- If $\left\{x_{n}\right\}$ tends to infinity, then $\left\{x_{n}\right\}$ is bounded below.

- If $\lim _{\mathrm{n} \rightarrow \infty} x_{n}= \pm \infty$, then $\left\{x_{n}\right\}$ is unbounded and increasing.

- If $\left\{x_{n}\right\}$ is unbounded and increasing, then $\lim _{\mathrm{n} \rightarrow \infty} x_{n}=\infty$.

- A sequence properly diverges if and only if it is unbounded.

In their subsequent discussion of the statements, students cited the non-monotone counterexamples by their animal names, pointing out that the second statement is false because of Platypus and the fourth statement "only works in one direction" because of Penguin.

Using this analogy, members of the class used the name Platypus to refer both to the sequence and to its role as an atypical example of a sequence that tended to infinity. In

\footnotetext{
${ }^{5}$ The class later defined that a sequence "tends to infinity," written $\lim _{n \rightarrow \infty} x_{n}=\infty$, if $\forall M>0, \exists \mathbf{N} \in \mathbf{N}$ such that $\forall n>\mathbf{N}, x_{n}>M$.
} 
contrast to prototypes, which are key examples that act as paradigms for or display the "standard" properties of a category, we use the term wedge to denote examples that help distinguish oft conflated categories. While students reasoning about categories in terms of familiar examples may overgeneralize certain properties, wedges help distinguish the two properties. The absolute value function is a standard wedge between continuity and differentiability. Wedges are a specific type of boundary example (Watson and Mason 2001). Platypus became the class' wedge between monotonicity and tending to infinity. The name Platypus, like the notion of a wedge, cites how exemplars can reflect on relationships between properties. The true/false questions emphasized that the example was pertinent to questions of "property implication," namely that Platypus is a counterexample to the possible theorem "If $\lim _{n \rightarrow \infty} x_{n}=\infty$, then $\left\{x_{n}\right\}$ is monotone."

About 2 weeks after the Penguin and Platypus analogies were introduced, one of the authors asked Vincent, a student in RA1, to recall the key sequences from class. He was able to recall both sequences and that one was "a bird" and the other was "the weird looking platypus." The interviewer asked him why the one sequence was a bird and he explained, "Cause we were talking about mammals which was... proper divergence." Earlier, he had stated his (nonstandard) personal concept definition (PCD; Tall and Vinner 1981) of proper divergence saying: "For every $K \in \mathbf{N}$... pick some term $x_{K}$ right here, then for every term... $n>K, x_{n}>x_{K}$. So all the $x_{n}$ 's got to be in that interval $\left[\left(x_{K}, \infty\right)\right]$ here." When asked to explain why the first sequence was a bird, Vincent said:

Vincent: This one here where every $x_{n}$ is going to be in this little interval right here... Say $x_{K}$ was 2 . Well you can go all the way over here, there is going to be a 1 which is not in this interval because it's less than $x_{K}$ which is 2 . So that gives us trouble so that doesn't properly diverge... Then this Platypus... if I pick an even term, it will work. If I pick an odd term, then I'm having trouble cause the term next to it is going to be less than it. But if I choose an even term, then every term after that is going to be greater than that so it does properly diverge.

Int.: What was interesting about this case that made it kind of funny?... Why do we label this one a platypus?

Vincent: Cause it wasn't exactly increasing all the way I guess. Cause it would kind of like go up down up down up down, that kind of deal, I guess... When people normally hear "tends to infinity," they might automatically think that the sequence is I guess monotonic, just increases, cause that's what I thought before she brought this up.

Vincent's PCD differed from the class' ratified definition of proper divergence. His definition seemed to entail monotonicity, because he claimed that the condition " $\forall n>K, x_{n}>x_{K}$ " should hold for every $K \in \mathbf{N}$. As such, he had to modify his definition to accommodate Platypus because only even-indexed terms "will work." Vincent knew that Platypus represented a strange example of proper divergence. He was also consciously aware that his understanding of tending to infinity entailed monotonicity. Vincent acknowledged that Dr. B used this example to target a misconception that he held. Why was Vincent certain that Platypus did properly diverge if it conflicted both 
with his concept image and his PCD? We cannot justify that Vincent's certainty came only from the Platypus analogy, but we find it plausible that naming the example according to its MM role helped Vincent understand how it was classified.

During an interview 1 month after that, the interviewer invited Vincent to "tell me all you can about" several example sequences he provided. He first presented the Penguin sequence written as " $1,1,2,1,3,1,4,1,5,1, \ldots$ " After listing several properties of the sequence, he noted "this is Penguin." When asked about the name, he knew that this was a non-example of a category because it was "not a mammal," but he initially claimed that mammals were convergent sequences.

After two other examples, the interviewer presented the Platypus sequence (" $2,1,4,3,6,5,8,7 \ldots$ ".), to which Vincent applied the analogical name before listing its properties. Vincent explained, "He is a weird looking mammal, or in this case a weird looking convergent sequence." However, as he tried to explain the analogy with convergence, he revealed that he was thinking about his PCD of tending to infinity:

Because we can find a $K$, or we can find a point in this sequence to where every point afterwards would be greater than it. Like, if I were to pick this point [pointing to 4] it wouldn't work because 3 is going to be less than it, but if I were to pick... an even [indexed] sequence in this case it would work out.

Vincent again displayed that his PCD differed from the class' definition because it conflated the bounds in the definition of proper divergence ( $M$ such that $x_{n} \geq M$ ) with the first term satisfying the condition upon the indices $\left(x_{K}\right.$ such that $x_{n} \geq x_{K}$ for all $\left.n>K\right)$. Dr. B introduced Platypus to address this exact misconception in the definition, but Vincent accommodated the example by restricting the reference of the definition to even-indexed terms.

Vincent then revised his analogy saying that mammals properly diverged. He said:

Vincent: When we were talking about mammals, we were talking about something that properly diverges... [Platypus] properly diverges. These [other examples] all here didn't properly diverge, so they were birdies [...] So, yeah, that's what our mammal was. It was properly divergent, not convergent. Sorry.

Int.: What is the relationship between properly divergent and monotonic?

Vincent: Hmm. I am not sure now.

Int:: Why do you say that? Why do you say that you are not sure now?

Vincent: When I was thinking monotonic, I was thinking every number in the sequence is going to be greater than, or the number next to it is going to be greater than it. That would make it a monotonic sequence, which makes it properly diverging... I am not sure about this one because this one diverges for sure, but the numbers next, like there is going to be a number in the sequence next to a certain number that's not bigger than [it]. Like I can pick this number and I'll say it will diverge from here, but every two terms the term next to it is going to be less than it. So now I am not sure anymore. I know it is divergent though. 
Vincent revised his analogical mapping between animals and categories of sequences when it could not accommodate the Platypus sequence. He returned to the analogy Dr. B used 6 weeks prior. His reflection revealed that his view that properly divergent sequences being monotonic persisted, but his classification of Platypus led him to question this. Again, his concept image and PCD both entailed monotonicity, but it seems that the Platypus analogy helped him recreate the reasoning by which the example disproved the property implication "If $\lim _{n \rightarrow \infty} x_{n}=\infty$, then $\left\{x_{n}\right\}$ is monotone."

\section{Comments on the Penguin and Platypus Analogies}

As in the previous cases, Dr. B was aware that students tended to harbor misconceptions regarding the classification of sequences. As was outlined previously, students are faced with simultaneously learning mathematical definitions themselves and the MM conventions for interpreting and using these definitions to classify and prove. Students tend to reason in terms of prototypes or sets of familiar examples, which can lead to overgeneralization (Alcock and Simpson 2002) or monster-barring (Lakatos 1976; Larsen and Zandieh 2008). Vincent was primed to fall into this pattern of reasoning regarding sequences that tend to infinity because both his concept image and PCD entailed monotonicity. However, on multiple occasions the Platypus sequence helped him to reflect on the relationship between the properties and revise his view that sequences tending to infinity are monotone. While Vincent represents a single case, he portrays how Dr. B used the analogy to draw students' attention to Platypus' role as a wedge example. Because Vincent elaborated the Platypus analogy and was able to correct it when misremembered, we can claim that the analogy did not merely serve a naming function. However, it usefully induced a language for discussing example categorization and property implication. As students referred to the mathematical object as a Platypus, they simultaneously named the object and cited its role in their defining/classifying activity. Penguin similarly served as a wedge between the properties $\lim _{n \rightarrow \infty} x_{n}=\infty$ and unbounded above.

Like the previous examples, the Penguin and Platypus analogies entailed a more implicit metaphor that compared mathematical classification to biological taxonomy. As the analogies drew students' attention to classification and defining, the metaphor suggested a way of reasoning about the classificatory process at hand (i.e. conventions of classifying and defining). Like White Tigers, the biological metaphor within the Platypus analogy evoked a sense that the example space was inexhaustible. One may encounter strange entities like a Platypus that cause problems for simple classification schemes (i.e. problematizing defining). As in her other defining activities, Dr. B emphasized how the definition needed to be able to capture all of the pertinent examples and exclude the others (Dawkins 2014).

The activities Dr. B introduced further aided students in perceiving the nature of mathematical classification and its conventions. As outlined above, the game of mathematical classification is organized around principles of inference. Classes must be well-defined by a stipulated definition to afford property implication and proof. The value of the Platypus example is not for deciding whether a single sequence does or does not tend to infinity, the mathematical question is whether, "If a sequence tends to infinity, then it is monotone." Viewed as an open statement (Durand-Guerrier 2003), it 
is true that many such sequences are monotone. However, under the mathematical convention of closed, general statements, Platypus alone is sufficient to render the claim "If $\lim _{n \rightarrow \infty} x_{n}=\infty$, then $\left\{x_{n}\right\}$ is monotone" false. We find that the Platypus and Penguin analogies helped students interpret these examples in ways more compatible with the conventions of mathematical practice regarding defining and relating properties.

\section{Discussion and Implications}

In this paper, we first set forth arguments based in previous research that MM and ML issues are 1) fundamental to proof-oriented mathematical practice, 2) problematic for students learning such mathematics, and 3) difficult to teach alongside particular mathematical content. Accordingly, we argued that instruction in ML and MM reasoning required means by which students could both be made aware of these mathematical issues and be given tools for reflecting and communicating about important conventions. We then provided three examples of how analogies were fruitfully used in real analysis classrooms to apprentice students into MM and ML reasoning. We present these examples as paradigms of an instructional method in proof-oriented mathematics classes. In what follows, we shall examine further why these analogies proved useful in light of our theoretical framing of the learning issues at hand. We also reflect further on the nature of the MM and ML framing of these instructional issues and its importance for mathematics education research and practice.

\section{Analogy/Metaphor Pairs}

As we observed in our survey of prior research, mathematical practice relies on particular conventions of practice that afford mathematics' claims to generality and justification. First, mathematicians try to use language in precise and unambiguous ways by closely corresponding syntactic forms with semantic meanings. Recognized ambiguities often prompt disambiguation of mathematical language. In this way, mathematicians train themselves (and each other) to consistently monitor their own language use to maintain the community's standards of discourse. Students must be apprenticed into such ML reasoning and into the conventions that mathematicians have adopted to solve language problems. Because a mathematical text is often understood as a trail of inference, mathematicians pay careful attention to how mathematical information epistemically unfolds over the course of the text. This often allows or disallows dependence among various mathematical objects in the text. To imagine the text unfolding in time induces a useful metaphor relating temporal order in text to the chronological order of events.

Before the students in RA2 conceived of multiple interpretations for multiply quantified statements, there was no disambiguation to be dealt with. The students were not fully aware of the problem implicit in Bill's mathematical argument. The two voices in the Mayan stonecutter story helped make the two interpretations perceivable. However, to make a disambiguating choice between the interpretations, students needed some way to choose an interpretation. The metaphor between time and order 
quickly and easily prompted them to side with the craftsman's interpretation, and then to see why Bill's argument was comparable to the priest's. Since the Mayan stonecutter story contrasted two interpretations of a linguistic form, embedding them in time, students recognized how dependence and independence (induced by the time metaphor) distinguished the two interpretations. Like the activities advocated by Epp (2003) and Durand-Guerrier (2003, 2008), the analogy harnessed students' compatible everyday understanding (of time in this case) to help them perceive mathematical convention and its utility for disambiguation. Neither do we claim that the students elaborated all these aspects of the analogy/metaphor, nor did they need to. The students in a sense saw the issue at hand (order and dependence) through the analogy. The key is that the analogy helped the students adopt mathematical conventions of interpretation by disambiguating distinct meanings.

The White Tigers, Penguin, and Platypus analogies similarly portray this analogy/ metaphor pair structure. While the Tigers-analogy mapped sets and bijections onto forests and white tigers, the implicit metaphor compared mathematical spaces to forests portraying the inexhaustibility of example spaces. It further portrayed how nonexamples of a property do not constitute proofs of the non-existence of exemplars with the property. Students seemed able to see standards of proof and disproof through the lens of the analogy. The success of the metaphor depended upon portraying the set of tigers (functions) as an inexhaustible space over which the statements were quantified, consistent with mathematical interpretations of quantification over infinite sets of examples.

The names Penguin and Platypus drew attention to the role of particular examples in relating categories of sequences, specifically properties that could be easily conflated. The analogy mapped a sequence, tending to infinity, and monotonicity onto the platypus, mammals, and having live young, respectively. The implicit metaphor compared mathematical classification to biological taxonomy and the desire for inclusive categories even when boundary examples do not fit prototypical images. The mathematical issue at hand is the extensivity of mathematical reference (inferences are assumed to be quantified over entire classes of objects by closed, general statements) and the stipulated nature of mathematical definitions. Categories must be understood to refer to any member of the category (not just prototypes) and odd examples cannot be rejected simply because they are counterintuitive (monster-barring). In fact, mathematicians often value examining odd examples most because they help test the bounds of what general theorems might be provable (Weber 2008). Students' ability to assimilate Platypus into their conception of tending to infinity constituted a valuable step in progressive generalization of the category. Platypus was thus valuable not only because it helped expand students' concept image of sequences that tend to infinity (Mason and Watson 2008), but also because it disproved the possible theorem "If $\lim _{\mathrm{n} \rightarrow \infty} x_{n}=\infty$, then $\left\{x_{n}\right\}$ is monotone."

In this way, the class' use of the metaphor not only affected Vincent's understanding of the example, but his view of how examples reflect relationships between categories. While a number of studies on students' use of examples call for greater attention to example-based reasoning in proof-oriented mathematics (e.g. Bills and Watson 2008; Lockwood et al. 2012), we perceive that students' use of examples in this context must be oriented toward their reflection on the relationships between categories and 
properties. In other words, students' focus must be shifted from classification or description (example-oriented) toward defining and implication (property-oriented). Students should see how particular examples reflect on generalized relations. We think the analogy helped shift Vincent's attention because the particular case was referred to by its role in relating categories, i.e. as a wedge example. Figure 2 portrays this shift.

\section{Attending to ML and MM Instruction}

In this paper, we drew together a number of teaching and learning issues under the heading of ML and MM practice. We find a strong link between these different issues both because of their inherently reflective nature (i.e. metareasoning), and because of the value we found in analogies for instruction. In each case, we observed how mathematicians adopt key conventions that help them maintain high standards of precision, clarity, and generality in their reasoning, communicating, and proving. Specifically, mathematicians induce dependence and independence through temporal order, much like chronological order (the Mayan stonecutter story). Mathematicians understand statements to be quantified over sets of examples (White Tigers), and statements are closed in the sense that a single counterexample is enough to negate a statement quantified over a set (Penguin \& Platypus).

The reader likely noted that many of the topics we describe in this way fall under the category of what has traditionally been called mathematical logic. We purposefully downplay this characterization of these teaching and learning matters because we perceive that much previous literature on "logic" tends to conflate descriptive and prescriptive models thereof (Dawkins 2014; Oaksford et al. 2012; Stenning and Monaghan 2004; Toulmin 1958). In other words, many do not view these conventions of mathematical practice as metaphors or choices (descriptive of practice), but as essentially correct meanings for language (prescriptively accurate). We adopt the ML and MM frame because it draws attention to the role of students' reasoning about their own language and reasoning in learning proof-oriented mathematics. As Stenning

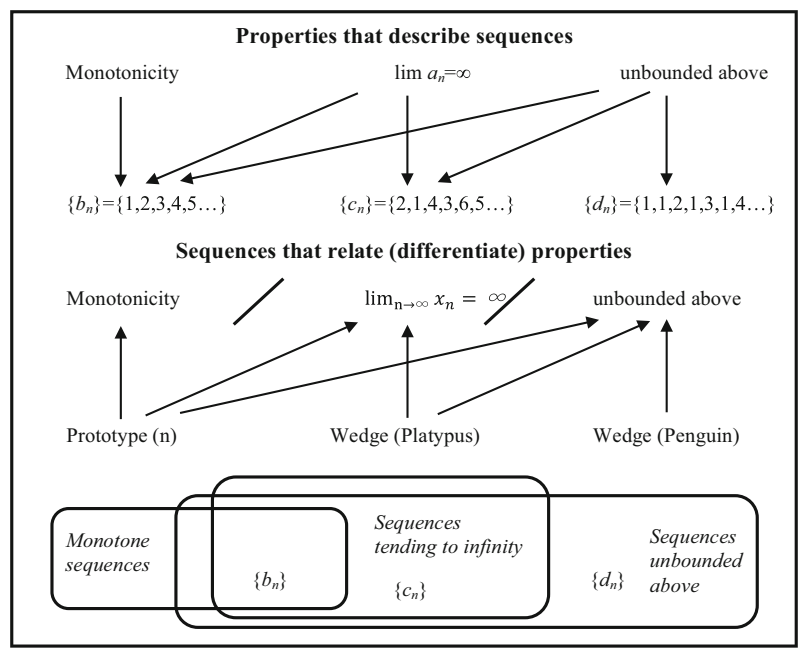

Fig. 2 Shifting attention from example descriptions to property relations 
(2002) points out regarding the psychological literature on logic, researchers often pursue fundamental aspects of human cognition such as internal representations or internal logics when matters of strategic competence, metareasoning, and learning may be far more pertinent and fruitful. We propose this way of framing learning logic and notation because it is much more compatible with a constructivist view of learning (Piaget 1950; Von Glasersfeld 1995). We offer analogies as a powerful methodological tool for helping students in the proof-oriented classroom and we provide the analogy/ metaphor pair as a theoretical frame for explaining their efficacy. Adopting this analytical lens entails framing the teaching and learning issues at hand in terms of student reflection upon their own language and reasoning, which we anticipate will prove useful for further mathematics education research and instruction. Stated another way, we endeavor to draw attention to the central importance of students' awareness of ML and MM issues, which are often implicit in proof-oriented mathematical discourse. Recasting logic and notation as metareasoning embeds them in the realm of student activity rather than mathematical abstraction - yet another product to process shift - which we perceive is necessary for the development of appropriate pedagogy for proof-oriented mathematics courses and for fruitful research on related teaching and learning phenomena.

\section{References}

Alcock, L., \& Simpson, A. (2002). Definitions: dealing with categories mathematically. For the Learning of Mathematics, 22(2), 28-34.

Antonini, S. (2011). Generating examples: focus on processes. ZDM, 43, 205-217.

Bills, L., \& Watson, A. (2008). Editorial introduction. Educational Studies in Mathematics, 69, 77-79.

Brousseau, G. (1997). Foundations and methods of didactique. In N. Balacheff, M. Cooper, R. Sutherland, \& V. Warfield (Eds.), Theory of didactical situations in mathematics: Didactique des Mathematiques 19701990 (pp. 21-75). Dordrecht, The Netherlands: Kluwer (Original work published 1986).

Burn, B. (2005). The vice: some historically inspired and proof-generated steps to limits of sequences. Educational Studies in Mathematics, 60, 269-295.

Copi, I. (1954). Symbolic logic. New York: The Macmillan Company.

Cory, B., \& Garofalo, J. (2011). Using dynamic sketches to enhance preservice secondary mathematics teachers' understanding of limits of sequences. Journal for Research in Mathematics Education, 42, 65-96.

Cottrill, J., Dubinsky, E., Nichols, D., Schwingendorf, K., Thomas, K., \& Vidakovic, D. (1996). Understanding the limit concept: beginning with a coordinated process scheme. The Journal of Mathematical Behavior, 15, 167-192.

Dahlberg, R., \& Housman, D. L. (1997). Facilitating learning events through example generation. Educational Studies in Mathematics, 33, 283-299.

Dawkins, P. (2009). Concrete metaphors in the undergraduate real analysis classroom. In Swars, S. L., Stinson, D. W., \& Lemons-Smith, S. (Eds.), Proceedings of the 31st Annual Meeting of the North American Chapter of the International Group for the Psychology of Mathematics Education (pp. 819-826). Atlanta: Georgia State University.

Dawkins, P. (2012). Metaphor as a possible pathway to more formal understanding of the definition of sequence convergence. The Journal of Mathematical Behavior, 31(3), 331-343.

Dawkins, P. C. (2014). How students interpret and enact inquiry-oriented defining practices in undergraduate real analysis. The Journal of Mathematical Behavior, 33, 88-105.

Dawkins, P., \& Roh, K. (2011). Mechanisms for scientific debate in real analysis classrooms. In Weist, L. \& Lamberg, T. (Eds.), Proceedings of the 33rd Conference of the North American Chapter of the International Group for the Psychology of Mathematics Education (pp. 820-828). Reno: University of Nevada, Reno. 
De Villiers, M. (1998). To teach definitions in geometry or to teach to define? In Olivier, A. \& Newstead, K. (Eds.), Proceedings of the 22nd Annual Meeting of the International Group for the Psychology of Mathematics Education (2, pp. 248-255). Stellenbosch: University of Stellenbosch.

Dubinsky, E., \& Yiparaki, O. (2000). On student understanding of AE and EA quantification. Research in Collegiate Mathematics Education, IV, 239-289.

Durand-Guerrier, V. (2003). Which notion of implication is the right one? From logical considerations to a didactic perspective. Educational Studies in Mathematics, 53(1), 5-34.

Durand-Guerrier, V. (2008). Truth versus validity in mathematical proof. ZDM, 40(3), 373-384.

Durand-Guerrier, V., \& Arsac, G. (2005). An epistemological and didactic study of a specific calculus reasoning rule. Educational Studies in Mathematics, 60(2), 149-172.

Durand-Guerrier, V., Boero, P., Douek, N., Epp, S. S., \& Tanguay, D. (2012). Examining the role of logic in teaching proof. In G. Hanna \& M. De Villiers (Eds.), Proof and proving in mathematics education (pp. 369-389). Netherlands: Springer.

Edwards, B., \& Ward, M. (2008). The Role of mathematical definitions in mathematics and in undergraduate mathematics courses. In M. Carlson \& C. Rasmussen (Eds.), Making the connection: Research and teaching in undergraduate mathematics education MAA Notes \#73 (pp. 223-232). Washington, DC: Mathematics Association of America.

Eisenberg, T., \& Dreyfus, T. (1994). On understanding how students learn to visualize function transformations. In E. Dubinsky, A. Schoenfeld, \& J. Kaput (Eds.), Research in collegiate mathematics education I (Vol. 4, pp. 45-68). Washington, DC: Mathematics Association of America.

Epp, S. (2003). The role of logic in teaching proof. The American Mathematical Monthly, 110, 886-899.

Epp, S. (2009). Proof issues with existential quantification. In F.-L. Lin, F.-J. Hsieh, G. Hanna, \& M. de Villiers (Eds.), Proceedings of ICMI Study 19: Proof and proving in mathematics education, Vol. 1 (pp. 154-159), Taipei, Taiwan.

Evans, J. (2005). Deductive reasoning. In K. J. Holyoak \& R. G. Morrison (Eds.), Cambridge handbook of thinking and reasoning (pp. 169-184). Cambridge: Cambridge University Press.

Font, V., \& Contreras, A. (2008). The problem of the particular and its relation to the general in mathematics education. Educational Studies in Mathematics, 69, 33-52.

Freudenthal, H. (1973). Mathematics as an educational task. Dordrecht: D. Reidel Publishing.

Freudenthal, H. (1991). Revisiting mathematics education: The China lectures. Dordrecht: Kluwer.

Gerson, H., \& Bateman, E. (2010). Authority in an agency-centered, inquiry-based university calculus classroom. The Journal of Mathematical Behavior, 29, 195-206.

Grice, H. P. (1957). Meaning. Philosophical Review, 66, 377-388.

Guzman, M. (2002). The role of visualization in the teaching and learning of mathematical analysis. In Proceedings of the International Conference on the Teaching of Mathematics (at the Undergraduate Level). Hersonissos, Crete, Greece.

Jahnke, H. N. (2008). Theorems that admit exceptions, including a remark on Toulmin. ZDM, 40(3), 363-371.

Lakatos, I. (1976). Proofs and refutations. Cambridge: Cambridge University Press.

Larsen, S., \& Zandieh, M. (2008). Proofs and refutations in the undergraduate classroom. Educational Studies in Mathematics, 67, 205-216.

Lockwood, E., Ellis, A. B., Dogan, M. F., Williams, C., \& Knuth, E. (2012). A framework for mathematicians' example-related activity when exploring and proving mathematical conjectures. In Proceedings of the 34th Annual Meeting of the North American Chapter of the International Group for the Psychology of Mathematics Education (pp. 151-158).

Mariotti, M. A., \& Fischbein, E. (1997). Defining in classroom activities. Educational Studies in Mathematics, 34(3), 219-248.

Mason, J., \& Watson, A. (2008). Mathematics as a constructive activity: Exploiting dimensions of possible variation. In M. Carlson \& C. Rasmussen (Eds.), Making the connection: Research and teaching in undergraduate mathematics education MAA notes \#73 (pp. 191-204). Washington, DC: Mathematics Association of America.

Murphy, G., \& Hoffman, A. (2012). Concepts. In K. Frankish \& W. Ramsey (Eds.), The Cambridge handbook of cognitive science (pp. 151-170). New York: Cambridge University Press.

O'Connor, M. C., \& Michaels, S. (1993). Aligning academic task and participation status through revoicing: analysis of a classroom discourse strategy. Anthropology and Education Quarterly, 24, 318-335.

Oaksford, M., Chater, N., \& Stewart, N. (2012). Reasoning and decision making. In K. Frankish \& W. Ramsey (Eds.), The Cambridge handbook of cognitive science (pp. 131-150). New York: Cambridge University Press.

Ouvrier-Buffet, C. (2006). Exploring mathematical definition construction processes. Educational Studies in Mathematics, 63(3), 259-282. 
Piaget, J. (1950). The psychology of intelligence. New York: Routledge Classics.

Piatek-Jimenez, K. (2010). Students' interpretations of mathematical statements involving quantification. Mathematics Education Research Journal, 22(3), 41-56.

Pinto, M., \& Tall, D. (2002). Building formal mathematics on visual imagery: a case study and a theory. For the Learning of Mathematics, 22(1), 2-10.

Roh, K. H. (2008). Students' images and their understanding of definitions of the limit of a sequence. Educational Studies in Mathematics, 69(3), 217-233.

Roh, K. (2009). Students' understanding and use of logic in evaluation of proofs about convergence. In F.-L. Lin, F.-J. Hsieh, G. Hanna, \& M. de Villiers (Eds.), Proceedings of ICMI Study 19: Proof and proving in mathematics education, Vol. 2 (pp. 148-153), Taipei, Taiwan.

Roh, K. (2010). An empirical study of students' understanding of a logical structure in the definition of limit via the $\varepsilon$-strip activity. Educational Studies in Mathematics, 73, 263-279.

Roh, K., \& Lee, Y. (2011). The Mayan activity: a way of teaching multiple quantifications in logical contexts. Problems, Resources, and Issues in Mathematics Undergraduate Studies, 21, 1-14.

Savic, M. (2011). Where is the logic in student-constructed proofs? In S. Brown, S. Larsen, K. Marrongelle, \& M. Oehrtman (Eds.), Proceedings of the 14th Conference on Research in Undergraduate Mathematics Education (Vol. 2, pp. 445-456). Portland, OR.

Shipman, B. A. (2012). Determining definitions for comparing cardinalities. PRIMUS, 22(3), 239-254.

Shipman, B. A., \& Shipman, P. D. (2013). Simple yet hidden counterexamples in undergraduate real analysis. PRIMUS, 23(2), 111-120.

Stenning, K. (2002). Seeing reason: Image and language in learning to think. Oxford: Oxford University Press.

Stenning, K., \& Monaghan, P. (2004). Strategies and knowledge representation. In J. P. Leighton \& R. J. Sternberg (Eds.), The nature of reasoning (pp. 129-168). Cambridge: Cambridge University Press.

Stenning, K., \& van Lambalgen, M. (2004). A Little logic goes a long way: basing experiment on semantic theory in the cognitive science of conditional reasoning. Cognitive Science, 28, 481-529.

Stylianides, G. J., \& Stylianides, A. J. (2008). Proof in school mathematics: insights from psychological research into students' ability for deductive reasoning. Mathematical Thinking and Learning, 10, 103133.

Swinyard, C. (2011). Reinventing the formal definition of limit: the case of Amy and Mike. The Journal of Mathematical Behavior, 30, 93-114.

Swinyard, C., \& Larsen, S. (2012). Coming to understand the formal definition of limit: insights gained from engaging students in reinvention. Journal for Research in Mathematics Education, 43, 465-493.

Tall, D. O. (2013). How humans learn to think mathematically: Exploring the three worlds of mathematics. New York: Cambridge University Press.

Tall, D. O., \& Vinner, S. (1981). Concept image and concept definition in mathematics with particular reference to limits and continuity. Educational Studies in Mathematics, 12(2), 151-169.

Thompson, P. W. (2013). In the absence of meaning.... In K. Leatham (Ed.), Vital directions for research in mathematics education. New York: Springer.

Toulmin, S. (1958). The uses of argument. Cambridge: Cambridge University Press.

Vinner, S. (1991). The role of definitions in teaching and learning mathematics. In D. Tall (Ed.), Advanced mathematical thinking (pp. 65-81). Dordrecht: Kluwer.

Von Glasersfeld, E. (1995). Radical constructivism: A way of knowing and learning. London: Falmer Press.

Watson, A., \& Mason, J. (2001). Getting students to create boundary examples. MSOR Connections, 1, 9-11.

Weber, K. (2008). How mathematicians determine if an argument is a valid proof. Journal for Research in Mathematics Education, 39(4), 431-459. 